EPJ Web of Conferences 62, 07005 (2013)

DOI: $10.1051 /$ epjconf/20136207005

(C) Owned by the authors, published by EDP Sciences, 2013

\title{
Unifying fission, quasi-fission and the extra push
}

\author{
Neil Rowley ${ }^{1, a}$ and Saffidine Grar Nabila ${ }^{2}$ \\ ${ }^{1}$ Institut de Physique Nucléaire, UMR 8608, 91406 Orsay Cedex, France \\ ${ }^{2}$ Département des Sciences de la Matière, Institut de Sciences et Technologie, \\ Centre Universitaire de Bordj Bou Arreridj, 340000, Algeria
}

\begin{abstract}
Using the above-barrier fusion probabilities of Berriman and the appropriate extra-push energies, we re-investigate the quasi-fission dynamics of reactions leading to the compound nucleus ${ }^{220} \mathrm{Th}$. A consistent description is obtained in terms of the entrancechannel barriers that are known to determine the initial capture process. The different dynamical deformations that give rise to these barriers significantly influence the potentialenergy landscapes onto which the captured system is injected.
\end{abstract}

\section{Introduction}

For very heavy systems, the formation of evaporation residues (ER) via heavy-ion reactions is an extremely complicated process. The major steps of capture, compound-nucleus creation (fusion) and ER survival are summarised by the expression

$$
\sigma_{\mathrm{ER}}(E)=\frac{\pi}{k^{2}} \sum_{L}(2 L+1) T_{L}(E) P_{\text {fus }}(E, L, \ldots) W_{\text {sur }}\left(E^{*}, L\right) .
$$

- Here, $T_{L}$ is the transmission coefficient of the partial wave $L$ through the external Coulomb barrier into the composite system. These elements determine the capture cross section for the reaction.

- $P_{\text {fus }}$ is the probability that the captured system will then evolve and fuse to form an equilibrated compound nucleus $(\mathrm{CN})$. Thus $\left(1-P_{\text {fus }}\right)$ is the probability of quasi-fission $P_{\mathrm{QF}}$, that is, that the two captured fragments will reseparate without forming an equilibrated $\mathrm{CN}$.

- The quantity $W_{\text {sur }}\left(E^{*}, L\right)$ is the probability that the compound nucleus, formed at an excitation energy $E^{*}$, will survive true fission by decaying into the ER channels in question.

Of course for heavy systems (high $Z_{1} Z_{2}$ ) with collective internal degrees of freedom in the target and/or projectile, the capture stage is complicated by the existence of a broad "distribution" of Coulomb barriers $[1,2]$. Our aim in this contribution is to investigate whether the configurations corresponding to those barriers might also play a role in determining the probability of quasi-fission. The experimental $\tilde{\sigma}_{\mathrm{ER}}$ for the different reactions have been multiplied by the factors given in the legend in order to account for differing losses due to quasi-fission.

\footnotetext{
ae-mail: rowley@ipno.in2p3.fr
}

This is an Open Access article distributed under the terms of the Creative Commons Attribution License 2.0, which permits unrestricted use, distribution, and reproduction in any medium, provided the original work is properly cited. 


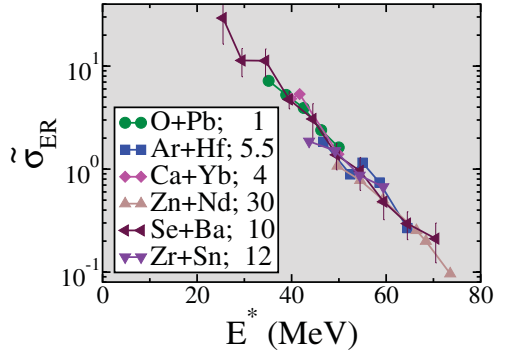

Figure 1. Above the energy of the Coulomb barriers, the reduced cross sections for the sum over the neutron-evaporation channels behave in the same way except for constant factors that depend on the entrance channel [5]. The experimental $\tilde{\sigma}_{\mathrm{ER}}$ for the different reactions have been multiplied by the factors given in the legend in order to account for differing losses due to quasi-fission.

Table 1. For the various target-projectile combinations studied, we show the Bass barrier $B$ [6], the excitation energy $E_{\mathrm{B}}^{*}$ when $E=B$, the symmetry parameter $\eta=A_{1} / A_{2}$ and the fusion probability at high-energy $P_{\text {fus }}$.

\begin{tabular}{lrcccr} 
Reaction & Ref. & $B(\mathrm{MeV})$ & $E_{\mathrm{B}}^{*}(\mathrm{MeV})$ & $\eta=A_{1} / A_{2}$ & $P_{\text {fus }}$ \\
\hline${ }^{16} \mathrm{O}+{ }^{204} \mathrm{~Pb}$ & {$[7]$} & 77.44 & 32.93 & 0.08 & $100 \%$ \\
\hline${ }^{40} \mathrm{Ar}+{ }^{180} \mathrm{Hf}$ & {$[8]$} & 145.2 & 45.72 & 0.22 & $18 \%$ \\
\hline${ }^{48} \mathrm{Ca}+{ }^{172} \mathrm{Yb}$ & {$[3]$} & 154.9 & 36.78 & 0.28 & $25 \%$ \\
\hline${ }^{70} \mathrm{Zn}+{ }^{150} \mathrm{Nd}$ & {$[9]$} & 196.5 & 38.57 & 0.47 & $3 \%$ \\
\hline${ }^{82} \mathrm{Se}+{ }^{138} \mathrm{Ba}$ & {$[10]$} & 206.6 & 26.07 & 0.59 & $10 \%$ \\
\hline${ }^{96} \mathrm{Zr}+{ }^{124} \mathrm{Sn}$ & {$[3]$} & 216.3 & 27.97 & 0.77 & $8 \%$ \\
\hline
\end{tabular}

\section{Obtaining $P_{\text {fus }}$ and the extra-push energies}

We shall consider only the sum over neutron-evaporation $(x-n)$ channels, since charged-particle channels are complicated by pre-equilibrium emission that significantly reduces the fissility of the lower- $Z$ residue [3]. The theoretical maximum production of $x$-n residues is determined by

$$
\tilde{\sigma}_{\max }\left(E^{*}\right)=\sum_{L}(2 L+1) W_{\text {sur }}\left(E^{*}, L\right)
$$

independent of the entrance-channel reaction. This unique "reduced cross section" will facilitate a comparison of different target-projectile combinations and permit some insights into the dynamics of quasifission. The channel-independence of this dimensionless quantity is assured by the Bohr hypothesis [4]; that is, the decay of an equilibrated $\mathrm{CN}$ depends only on its excitation energy $E^{*}$ and angular momentum $L$ and not on the manner in which it was formed. Having used $T_{L}=1$ in this expression guarantees that all of the angular momenta leading to ER creation are fully populated, making the expression depend solely on $E^{*}$. Thus Eq. (2) yields the maximum ER production possible, since it also takes $P_{\text {fus }}=1$. The cross section itself is restored by the factor $\pi / k^{2}$, introducing a trivial entrance-channel dependence, since the excitation energy and the incident energy $E$ are related through the reaction $Q$ value: $E^{*}=E+Q=E+\left(M_{1}+M_{2}-M_{\mathrm{CN}}\right) c^{2}$.

The relevance of the above result was demonstrated by Berriman et al. [5] for various reactions leading to the compound nucleus ${ }^{220} \mathrm{Th}$, and we shall study here the same systems listed in Table 1. Figure 1 demonstrates that above their Coulomb barriers, they all behave in the same way; that is, apart from a system-dependent factor (indicated in the legend), all of the ER cross sections are given by the same $\tilde{\sigma}_{\max }$. We make the following observations, that will be pursued in more detail elsewhere:

- the underlying $\tilde{\sigma}_{\max }$ can be well fitted by statistical-model (HIVAP) calculations, which are also capable of generating the individual $x$-n cross sections that are peaked at different excitation energies,

- by using the sum of these channels, $\tilde{\sigma}_{\max }$ is a relatively smooth function that can be seen from Figure 1 to describe the data over more than two orders of magnitude, and 


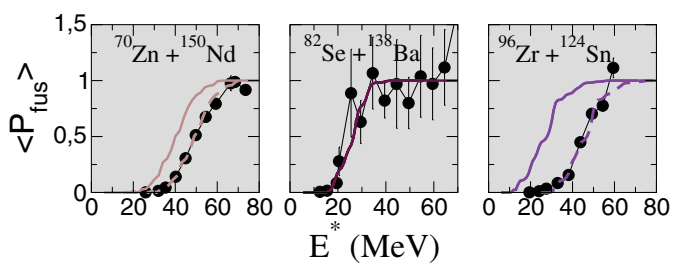

Figure 2. Fusion probabilities $\left\langle P_{\text {fus }}\right\rangle$ are shown normalised to their values at high energy for the three systems with the highest $Z_{1} Z_{2}$ values. Relative to $T_{0}$ (solid curves) the "extra push" is $9 \mathrm{MeV}$ for ${ }^{70} \mathrm{Zn}+{ }^{150} \mathrm{Nd}$ and $19 \mathrm{MeV}$ for ${ }^{96} \mathrm{Zr}+{ }^{124} \mathrm{Sn}$. There appears to be little shift for ${ }^{82} \mathrm{Se}+{ }^{138} \mathrm{Ba}$.

- much can be learned from lower energies where the individual systems deviate from $\tilde{\sigma}_{\text {max }}$.

The latter point is best shown through the fusion probability of Sahm et al. [3], whose seminal paper also provides the data on two of the systems displayed in Figure 1. These authors define an energy-dependent fusion probability from the experimental ER data

$$
<P_{\text {fus }}>=\tilde{\sigma}_{\mathrm{ER}} / \tilde{\sigma}_{\max }
$$

Of course at high energies this is equivalent to the result of Berriman et al. [5]. However, the important new idea stemming from Ref. [5] is to exploit a set of experimental systems to obtain an unambiguous, experimental $\tilde{\sigma}_{\max }$, defined between 25 and $75 \mathrm{MeV}$, a region over which it varies by more than two orders of magnitude. This is facilitated by the following:

- although the ${ }^{16} \mathrm{O}+{ }^{204} \mathrm{~Pb}$ system provides data only over a relatively small part of this energy range, it is an experimental fact (as well as a strong theoretical expectation) that $P_{\text {fus }}=1$ for this system, giving data points to which the other systems can be successively normalised.

- The data for ${ }^{82} \mathrm{Se}+{ }^{138} \mathrm{Ba}$ have large statistical errors but span almost all of the above range.

- The other systems provide convincing confirmation of the assertion in different parts of the range.

Furthermore, the excellent data on ${ }^{70} \mathrm{Zn}+{ }^{150} \mathrm{Nd}$ and ${ }^{96} \mathrm{Zr}+{ }^{124} \mathrm{Sn}$ extend well into the lower-energy region where $<P_{\text {fus }}>$ has not yet saturated, and demonstrate clearly the extra-push phenomenon. This is shown in Figure 2 where we have followed the earlier procedure of normalising $\left\langle P_{\text {fus }}>\right.$ to unity at higher energies. (In earlier works this was necessary since the only available $\tilde{\sigma}_{\text {max }}$ came from parameterdependent statistical-model calculations rather than the above experimental procedure.)

In order to obtain a value for the extra-push energy, one has to compare $\left\langle P_{\text {fus }}\right\rangle$ with the s-wave transmission $T_{0}$ for the penetration of the Coulomb barrier. For high $Z_{1} Z_{2}$ systems, the centrifugal energy is small for the relatively low $L$ values that survive fission, and so one might expect $<P_{\text {fus }}>$ to be similar to $T_{0}$ but shifted up slightly in energy. The solid curves in Figure 2 show the $T_{0}$ obtained from extensive coupled-channels calculations (CCFULL [11]) that include the important collective modes of both target and projectile. The shifts in $\left\langle P_{\text {fus }}\right\rangle$ for ${ }^{70} \mathrm{Zn}+{ }^{150} \mathrm{Nd}(9 \mathrm{MeV})$ and for ${ }^{96} \mathrm{Zr}+{ }^{124} \mathrm{Sn}(19 \mathrm{MeV})$ are in fact seen to be very substantial. In Figure 3, the solid curve (jagged due to pairing energies) shows $E_{\mathrm{B}}^{*}$ (the excitation energy for creation at the energy of the uncoupled barrier) for a wide range of targets and projectiles. The stable-stable combinations of interest here are indicated by the symbols defined in the legend. In addition we place a vertical bar through each symbol to indicate the range of the fully-coupled barrier distributions. The rest of our discussion relates to the three most symmetric systems of Figure 2 from which some interesting conclusions can be drawn.

It is tempting to associate the extra push for ${ }^{70} \mathrm{Zn}+{ }^{150} \mathrm{Nd}$ and for ${ }^{96} \mathrm{Zr}+{ }^{124} \mathrm{Sn}$, with the maximum in the $E_{\mathrm{B}}^{*}$ curve of Figure 3 (around $Z_{\text {projectile }}=16$ ), since this might appear to present a barrier to fusion when starting from more symmetric systems. The peak energy is around $45 \mathrm{MeV}$ and the observed extra-push energies put these systems above that. However, the behaviour for ${ }^{82} \mathrm{Se}+{ }^{138} \mathrm{Ba}$ is totally inconsistent with this explanation, since its $\left\langle P_{\text {fus }}\right\rangle$ saturates well below $45 \mathrm{MeV}$ (see Fig. 2). 


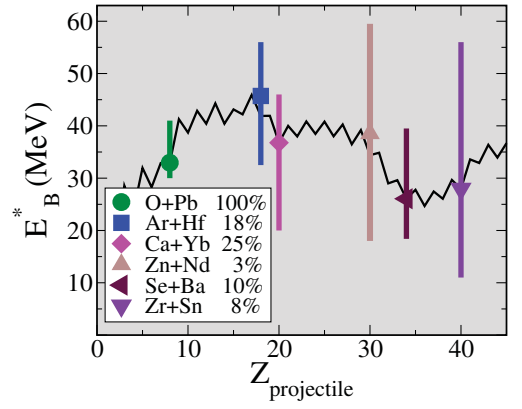

Figure 3. For many of the possible entrance-channel combinations leading to the ${ }^{220} \mathrm{Th}$ compound nucleus, the solid curve shows, as a function of projectile charge, the excitation energy $E_{\mathrm{B}}^{*}$ obtained for fusion at the energy of the uncoupled Coulomb barrier (using the Bass potential [6]). Six stable-stable combinations of target and projectile, for which measurements have been made, are highlighted (see Table 1). The bars indicate the energy range of the corresponding barrier distributions obtained by coupling to the collective modes of both target and projectile using CCFULL [11].

A major difference between ${ }_{34}^{82} \mathrm{Se}_{48}+{ }_{56}^{138} \mathrm{Ba}_{82}$ and the two adjacent systems is the closeness to neutron-shell closures in both target and projectile. An important consequence of this is the reduction of coupling effects on the barrier distribution. This can be seen from the shorter vertical bar for this system, and suggests a possible explanation of the differences between these three reactions in terms of their entrance-channel shape configurations. Indeed the effect of these configurations proves to be more significant than the corresponding shell-energy correction seen around $Z_{\text {projectile }}=36$ in Figure 3 .

\section{Potential-energy surfaces; $Z^{2} / A$, mass-asymmetry and deformation}

The evolution of the captured system toward the compound nucleus is generally accepted to be the result of thermal fluctuations upon a potential-energy surface that depends on the fragment separation and mass asymmetry (the thermal energy being provided by strong dissipation as the two partcipants lose kinetic energy on coming into contact). Of course other parameters such as the fragment deformation can also play a role. Indeed this is dominant in the fission process, where the two fragments become greatly elongated - an effect without which one cannot explain the total kinetic-energy systematics of Viola et al. [12]. Of course deformations can also exist in the entrance channel. This is obvious in the case of a target with a large static deformation, and in that case some consequences of the orientation and 'compactness' of the system have been discussed by Hinde et al. [13].

For vibrational systems, deformations are induced during the collision. Indeed they are responsible for the vast span of barriers $\left(10 \leq E^{*} \leq 55 \mathrm{MeV}\right)$ for the ${ }^{96} \mathrm{Zr}+{ }^{124} \mathrm{Sn}$ system. The higher barriers correspond to smaller separations of the centres of charge when the target and projectile surfaces come close together (compact configuration); conversely the lower barriers correspond to non-compact configurations with larger separations. Representing the ${ }^{96} \mathrm{Zr}$ and ${ }^{124} \mathrm{Sn}$ by charged ellipsoids, we find that the highest barrier essentially corresponds to an oblate deformation with an axis ratio $\varepsilon \approx 0.7$ and the lowest barrier corresponds to a prolate deformation with $\varepsilon \approx 1.3$. The relevant shapes are shown in Figure 4, where we also include a neck between the two nuclei; this neck is critical to the evolution of the system after touching. Before discussing deformations, we show in Figure 5 the potential energy surfaces (PES) for three different compound nuclei, all with spherical fragments. The central panel shows the compound nucleus ${ }^{220} \mathrm{Th}$ that we are currently considering. (The values of the potential along the touching curve are lower than the $E_{\mathrm{B}}^{*}$ of Figure 3, since the energy is strongly modified by the presence of a neck.) The left panel shows the lighter system ${ }^{170} \mathrm{Yb}$ and the right-hand panel shows the superheavy nuclide ${ }_{112}^{286} \mathrm{Cn}$. The PES is structured very differently in the three cases, due to the different fissility parameters $Z^{2} / A$. The nucleus ${ }^{170} \mathrm{Yb}$ is not far from the Businaro-Gallone point, and the potential is rather flat along the "touching" line. However, the potential is strongly driving towards fusion in the separation degree of freedom. In the ${ }^{220} \mathrm{Th}$ case, except for very small values of $A_{1} / A_{2}$ (for example, ${ }^{16} \mathrm{O}+{ }^{204} \mathrm{~Pb}$ ), the potential along the touching line drives towards symmetry where it is much 


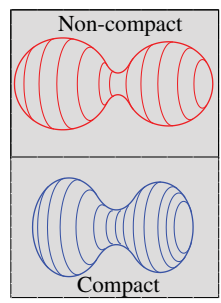

Figure 4. The potential-energy surfaces of Figures 5 and 6 are calculated with ellipsoidal shapes whose volumes reproduce those of the target and projectile nuclei. The axis ratio $\varepsilon$ is 1 for all panels of Figure 5. The shapes shown here correspond to the centre and left-hand panels of Figure 6, and represent approximately the deformations for the lowest-energy barrier $(\varepsilon=1.3$; prolate, non-compact) and the highest-energy barrier $(\varepsilon=0.7$; oblate, compact) for the ${ }^{96} \mathrm{Zr}+{ }^{124} \mathrm{Sn}$ reaction. Neck shapes are those at the corresponding scission points.
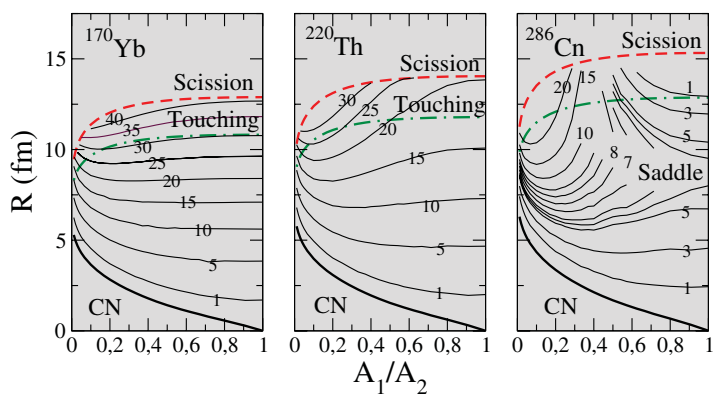

Figure 5. The horizontal axis gives the mass-symmetry of the fragments $\eta=A_{1} / A_{2}$, and the vertical axis their separation. Potential-energy surfaces (contours labeled in MeV) are shown for the compound nuclei ${ }^{170} \mathrm{Yb},{ }^{220} \mathrm{Th}$ and ${ }^{286} \mathrm{Cn}$, with both fragments treated as spherical. Table 1 gives initial values of $A_{1} / A_{2}$ for the ${ }^{220} \mathrm{Th}$ reactions.
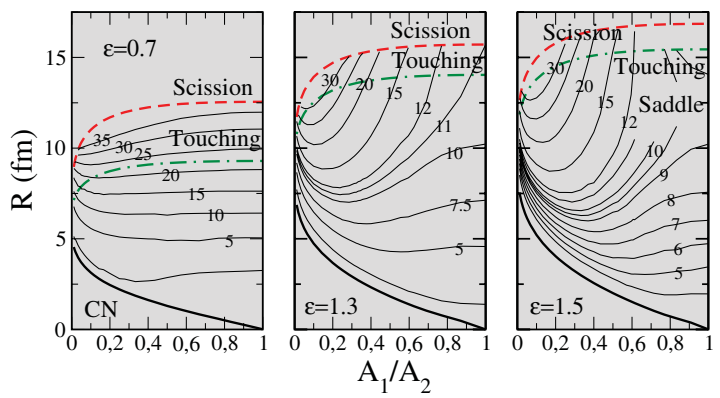

Figure 6. Potential-energy surfaces for ${ }^{220} \mathrm{Th}$ with three different deformations: $\varepsilon=0.7,1.3$ and 1.5 .

flatter in the $R$ direction. Thus fluctuations can easily lead to the quasi-fission of the system. For ${ }^{286} \mathrm{Cn}$, we find a fission barrier in the $R$ direction, and the probability of fusion will clearly be small.

Figure 6 again shows the PES for ${ }^{220} \mathrm{Th}$ but with three different deformations. The left-hand panel has $\varepsilon=0.7$, corresponding to the top of the barrier distribution for ${ }^{96} \mathrm{Zr}+{ }^{124} \mathrm{Sn}$. We see that this makes the PES structurally similar to that for the lighter system ${ }^{170} \mathrm{Yb}$ without deformation. Thus there is a much stronger driving force towards fusion for this deformation. Conversely, the central panel has $\varepsilon=1.3$ (bottom of the barrier distribution), and now the force driving to fusion has virtually vanished. The right-hand panel shows a slightly larger $\varepsilon=1.5$ for which the PES now resembles that for ${ }^{286} \mathrm{Cn}$ in Figure 5, possessing a saddle point with a barrier in the $R$ direction.

It is clear that the probability of fusion will be significantly higher for injection with the large oblate deformations that correspond to the highest part of the ${ }^{96} \mathrm{Zr}+{ }^{124} \mathrm{Sn}$ entrance-channel barrier distribution. And this will clearly give rise to a large extra-push effect. 


\section{Conclusions}

The structure of the liquid-drop potential-energy surface changes with $Z^{2} / A$, as the relative importance of Coulomb $\left(\propto Z^{2} / A^{1 / 3}\right)$ and surface $\left(\propto A^{2 / 3}\right)$ energies changes. For heavy symmetric systems, the Coulomb term begins to dominate for symmetric-mass partitions, leading to an increased probability for fission and quasi-fission. However, the balance between these two terms is restored for large oblate deformations. These can occur in the entrance channel for strongly vibrational systems such as ${ }^{96} \mathrm{Zr}+$ ${ }^{124} \mathrm{Sn}$, but only at the high-barrier end of the distribution. This results in a shift of the fusion probability to high energies - here, an extra-push energy of $19 \mathrm{MeV}$ with $P_{\text {fus }}=8 \%$. In the case of ${ }^{82} \mathrm{Se}+{ }^{138} \mathrm{Ba}$, the major effect of the closed neutron-shells appears to be a reduction in collectivity of the vibrational states. This gives a much less broad barrier distribution, so the system does not experience either extreme oblate or prolate deformations and there is little or no extra push. For the ${ }^{70} \mathrm{Zn}+{ }^{150} \mathrm{Nd}$ reaction, the target is deformed rather than vibrational, and the lack of axial symmetry (except for the very lowest barrier) makes a detailed calculation of the PES difficult. However, around the highest barrier (impact near the target equator) the gain in surface energy will clearly not be as great as for two participants that both possess an oblate deformation (see Fig. 4). In this case the extra-push energy is $9 \mathrm{MeV}$ and $P_{\text {fus }}$ is merely $3 \%$, despite the smaller initial value of $A_{1} / A_{2}$.

Apart from our capture-barrier distributions and the potential-energy surfaces for axially symmetric systems, the arguments presented here are largely qualitative. Nonetheless, even a cursory comparison of Figures 5 and 6 leads to the conclusion that the entrance-channel shape configurations of both target and projectile must be considered when calculating fusion probabilities for heavy systems. Here we have mainly considered vibrational nuclei, but a similar conclusion has been drawn by Nishio [14] for hot-fusion reactions with a deformed target.

\section{References}

[1] N. Rowley, G.R. Satchler and P.H. Stelson, Phys. Lett. B254, 25 (1991)

[2] M. Dasgupta, D.J. Hinde, N. Rowley and A.M. Stefanini, Ann. Rev. Nucl. Part. Sci. 48, 401 (1998)

[3] C.C. Sahm et al., Nucl. Phys. A441, 316 (1985)

[4] N. Bohr, Nature 137, 344 (1936)

[5] A.C. Berriman et al., Nature Vol. 413, 144 (2001)

[6] R. Bass, Phys. Rev. Lett. 39, 265 (1977)

[7] D.J. Hinde, M. Dasgupta and A. Mukherjee, Phys. Rev. Lett. 89, 282701 (2002)

[8] D. Vermeulen et al., Z. Phys. A318, (1984) 157

[9] C. Stodel, PhD thesis, LPC Caen (1998)

[10] K. Satou et al., Phys. Rev. C65, (2002) 054602

[11] K. Hagino, N. Rowley and A.T. Kruppa, Comp. Phys. Comm. 123, 143 (1999)

[12] V. E. Viola, K. Kwiatkowski and M. Walker, Phys. Rev. C31, 1550 (1985)

[13] D.J.Hinde et al., Phys. Rev. Lett. 74, 1295

[14] K. Nishio, contribution to this conference, and references therein 